\title{
Macrophage migration inhibitory factor induces autophagy to resist hypoxia/serum deprivation-induced apoptosis via the AMP-activated protein kinase/mammalian target of rapamycin signaling pathway
}

\author{
WENZHENG XIA ${ }^{1}$ and MENG HOU ${ }^{2}$ \\ Departments of ${ }^{1}$ Neurosurgery and ${ }^{2}$ Radiation Oncology, The First Affiliated Hospital, \\ Wenzhou Medical University, Wenzhou, Zhejiang 325000, P.R. China \\ Received February 11, 2015; Accepted October 23, 2015
}

DOI: $10.3892 / \mathrm{mmr} .2016 .4847$

\begin{abstract}
Macrophage migration inhibitory factor (MIF) is an anti-apoptotic agent in various cell types and protects the heart from stress-induced injury by modulating autophagy. Autophagy, a conserved pathway for bulk degradation of intracellular proteins and organelles, helps to preserve and recycle energy and nutrients for cells to survive during starvation. The present study hypothesized that MIF protects bone marrow-derived mesenchymal stem cells (MSCs) from apoptosis by modulating autophagy via the AMP-activated protein kinase/mammalian target of rapamycin (AMPK/mTOR) signaling pathway. MSCs were obtained from rat bone marrow and cultured. Apoptosis was induced by hypoxia/serum deprivation for $24 \mathrm{~h}$ and was assessed using flow cytometry. MIF protected MSCs from apoptosis by modulating autophagy via the AMPK/mTOR signaling pathway resulting in increased expression of autophagy-associated proteins (including LC3BI/LC3BII, Beclin-1 and autophagy protein 5), and by increased phosphorylation of AMPK and decreased phosphorylation of mTOR. The MIF anti-apoptotic effects were blocked by autophagy inhibitor, 3-methyladenine or AMPK inhibitor, Compound $\mathrm{C}$. These results indicate that MIF exerts a permissive role in protecting MSCs from apoptosis by regulation of autophagy via the AMPK/mTOR signaling pathway.
\end{abstract}

\section{Introduction}

Stem cell transplantation is a promising novel treatment strategy for ischemic cardiomyopathy, potentially resulting

Correspondence to: Dr Meng Hou, Department of Radiation Oncology, The First Affiliated Hospital, Wenzhou Medical University, 2 Fuxue Road, Wenzhou, Zhejiang 325000, P.R. China

E-mail:244517813@qq.com

Key words: macrophage migration inhibitory factor, mesenchymal stem cell, autophagy, AMP-activated protein kinase/mammalian target of rapamycin signaling pathway, apoptosis in cardiac repair and regeneration. Various different types of stem and progenitor cells are being investigated to assess their therapeutic effect (1). Mesenchymal stem cells (MSCs) are easily isolated and expanded, and exhibit low immunogenicity (2). However, their low survival rate after transplantation into damaged myocardium hinders their therapeutic potential. It has been demonstrated that the majority of donor MSCs, transplanted into the challenging microenvironment induced by acute myocardial infarction (AMI), undergo apoptosis in vivo. In vitro, hypoxia and serum deprivation (hypoxia/SD) are utilized to imitate the ischemic environment and have been shown to induce apoptosis in MSCs $(3,4)$. Therefore, improvement of MSC survival in an ischemic environment is a key challenge in developing MSC-mediated treatments in hearts following AMI.

Macrophage migration inhibitory factor (MIF) is a pleiotropic cytokine expressed in various cell types, including monocytes/macrophages, smooth muscle cells of the vascular system and cardiomyocytes (5-7). In addition, it acts as a regulatory factor for inflammation, apoptosis, autophagy, and glucose catabolism (8-11). Furthermore, MIF provides cardiac protection by promoting energy uptake under stress $(9,12)$. MIF has been identified as an anti-apoptotic agent in various cell types $(8,13)$, and has recently been demonstrated to protect cardiomyocytes from apoptosis by modulating autophagy under stress conditions (9). Autophagy is induced by MIF in conditions such as inflammation and starvation to counter the stress (14). Previous studies have indicated that MIF is involved in the maintenance of cardiac contractile function during starvation and protects cardiomyocytes from apoptosis by regulating autophagy (9). The above-mentioned studies demonstrate that MIF may also function in a protective capacity against hypoxia/SD-induced apoptosis in MSCs via autophagic regulation (15).

Autophagy is a catabolic process that maintains cellular homeostasis despite a variety of cellular stresses, including nutrient starvation, infection, damaged organelles and protein aggregates $(16,17)$. Autophagy and apoptosis are two major pathways utilized in a cell in response to stress. They are commonly co-regulated, but result in opposite cellular outcomes (18). Autophagy is a self-catabolic process where 
double-membrane vacuoles, called autophagosomes, form around components of the cell and degrade them utilizing lysosomal machinery (19). Autophagy is also critical in adaptive survival during periods of metabolic starvation or stress by maintaining nutrient availability and energy levels. By contrast, apoptosis is the self-elimination of a damaged or non-functional cell (20). Numerous studies have identified that stressors may trigger either process depending on the cellular context $(21,22)$. Inhibition of either pathway, by genetic or chemical methods, results in the activation of the other; blocking apoptosis in cells that would usually die triggers autophagy and survival, whereas blocking induction of autophagy in cells that would usually survive rapidly induces apoptosis $(23,24)$. Recent studies have confirmed that autophagy has a protective effect on MSCs under stress conditions (25). Therefore, modulation of autophagy may provide a novel mechanism to prevent apoptosis in MSCs under stress conditions.

Notably, previous studies have demonstrated the AMP-activated protein kinase/mammalian target of rapamycin (AMPK/mTOR) signaling pathway is activated by MIF to regulate autophagy and protect cells from apoptosis $(9,13,26,27)$. In hypoxia/SD, or other energy shortage situations, AMPK may sense the cellular energy change and become activated by a decreased ATP/AMP ratio, thus indirectly activating mTOR, one of its major downstream targets (9). Activation of the AMPK/mTOR signaling pathway may in turn stimulate autophagy and exert an anti-apoptotic effect (15). The AMPK/mTOR signaling pathway has been demonstrated to exert a protective role in MSCs $(15,28)$. Thus, activation of the AMPK/mTOR signaling pathway in MSCs may be a positive regulator of autophagy resulting in MSCs that are resistant to hypoxia/SD-induced apoptosis.

\section{Materials and methods}

Animals. Male Sprague Dawley rats, weighing 60-80 g, were cared for in accordance with the U.S. National Institutes of Health (NIH) guidelines (29). All of the study procedures were approved by the Wenzhou Medical University Institutional Animal Care and Use Committee (Wenzhou, China). Furthermore, the present study was conducted in compliance with the Guide for the Care and Use of Laboratory Animals, published by the National Academy Press (NIH; revised 1996). Ethical approval was obtained from Wenzhou Medical University Ethical Research Committee. The mice were raised in the Wenzhou Medical University Laboratory Animals Center, and raised separately, and fed with nourishment supplied by Research Diets, Inc. (New Brunswick, NJ, USA). The animals were housed in a light/dark cycle of $12 \mathrm{~h}$ light/12h dark at $20-25^{\circ} \mathrm{C}$.

Reagents. Dulbecco's modified Eagle's medium (DMEM) and fetal bovine serum (FBS) were obtained from GE Healthcare Life Sciences (Hyclone; Logan, UT, USA). The Annexin V-fluorescein isothiocyanate (FITC) Apoptosis Detection kit was obtained from BD Pharmingen (San Diego, CA, USA). Rabbit anti-rat monoclonal antibodies, LC3BI/LC3BII (1:1,000; cat. no. 3868), Beclin-1 (1:750; cat. no. 3495), autophagy protein 5 (Atg5; 1:1,000; cat. no. 5831), AMPK (1:1,000; cat. no. 5831), phosphorylated (p)-AMPK (1:500; cat. no. 2325), p-mTOR (1:500; cat. no. 2971) and mTOR (1:1,000; cat. no. 2972) were purchased from Cell Signaling Technology, Inc. (Danvers, MA, USA). Mouse polyclonal antibody anti- $\beta$-actin (1:1,000; cat. no. TA-09) was purchased from Beijing Zhongshan Goldenbridge Biotechnology, Co., Ltd. (Beijing, China). Anti-mouse/anti-rabbit secondary antibody horseradish peroxidase conjugate (1:1,000; cat. no. sc-2954) were obtained from Santa Cruz Biotechnology, Inc. (Dallas, TX, USA) and 3-methyladenine (3-MA) was obtained from Sigma-Aldrich (St. Louis, MO, USA). AMPK inhibitor, Compound C was obtained from Merck Millipore (Darmstadt, Germany). Rat recombinant MIF was obtained from Prospec-Tany TechnoGene, Ltd. (East Brunswick, NJ, USA).

Cell culture and treatment. Bone marrow-derived (BM)-MSCs were isolated from the femurs and tibias of Sprague-Dawley rats, as described in a previous study (30). Bone marrow cells were flushed from the femurs and tibias with $5 \mathrm{ml}$ DMEM/F12 medium (Gibco; Thermo Fisher Scientific, Inc., Waltham, MA, USA). After the red blood cells were lysed with red cell lysis buffer (Beyotime Institute of Biotechnology, Inc., Jiangsu, China) and removed, $5 \times 10^{5}$ cells were seeded in a $25-\mathrm{cm}^{2}$ flask with $6 \mathrm{ml}$ DMEM/F12 supplemented with $10 \%$ FBS and $1 \% 0.06 \mathrm{~mL}$ penicillin/streptomycin, (Beyotime Institute of Biotechnology, Inc.) and incubated at $37^{\circ} \mathrm{C}$ with $5 \% \mathrm{CO}_{2}$. Following three days of culture, non-adherent cells were removed, the medium was replaced and adherent MSCs were grown in the medium, which was replaced every three days. Upon reaching 80-90\% confluence, adherent cells were trypsinized (Beyotime Institute of Biotechnology, Inc.) and expanded at 2:3 or 1:2 dilutions. All of the cells used in the assay were from passages three to five.

Induction of apoptosis in vitro by hypoxia/SD, which was designed to mimic the in vivo conditions of the ischemic myocardium, was initiated, as described in a previous study (4). Cells exposed to hypoxia/SD alone served as apoptotic controls, with hypoxia induced by incubating MSCs in serum-free media in a glove box (Plas Labs 855-AC; Plas Labs, Inc., Lansing, MI, USA) under a controlled anaerobic atmosphere at $37^{\circ} \mathrm{C}$ to scavenge free oxygen. MIF (100 ng/ml) was added to the medium at the start of the hypoxia/SD exposure and incubation continued for $24 \mathrm{~h}$ under hypoxic conditions. The concentration of MIF used in the current study was based on the concentration administered in previous studies $(8,13)$.

Cells were preincubated with $10 \mathrm{mmol} / \mathrm{l}$ AMPK inhibitor, Compound $\mathrm{C}$ or $5 \mathrm{mmol} / \mathrm{l}$ autophagy inhibitor, 3-MA in complete medium under normoxic conditions for $90 \mathrm{~min}$. MIF $(100 \mathrm{ng} / \mathrm{ml})$ was then added to the cultures and the cells were placed under conditions of hypoxia/SD for $24 \mathrm{~h}$.

Flow cytometric analysis of cell apoptosis. Apoptosis was determined by detecting phosphatidylserine on cell membranes using the fluorescent dye, Annexin V-FITC Apoptosis Detection kit (BD Pharmingen), according to the manufacturer's protocols. This assay differentiates between intact [Annexin $\mathrm{V}^{-}$/propidium iodide $(\mathrm{PI})^{-}$], early apoptotic (Annexin $\left.\mathrm{V}^{+} / \mathrm{PI}^{-}\right)$, late apoptotic $\left(\right.$Annexin $\mathrm{V}^{+} / \mathrm{PI}^{+}$) and necrotic (Annexin $\mathrm{V}^{-} / \mathrm{PI}^{+}$) cells. Cells were harvested and washed three times in ice-cold phosphate-buffered saline (PBS; Beyotime Institute of Biotechnology, Inc.), resuspended in $300 \mu \mathrm{l}$ binding buffer and incubated with $5 \mu$ l Annexin V-FITC solution 
for $30 \mathrm{~min}$ at $4^{\circ} \mathrm{C}$ in the dark. This was followed by further incubation with $5 \mu \mathrm{l}$ PI for $5 \mathrm{~min}$ and immediate analysis by bivariate flow cytometry using the BD FACSCantoII equipped with FACSDiva software (642218 Rev.A; BD Biosciences, Franklin Lakes, NJ, USA). Approximately $1-5 \times 10^{5}$ cells were analyzed in each experiment.

Toxicity assay. The potential toxicity of MIF at varying concentrations was investigated in cultured MSCs. MSCs were incubated for $24 \mathrm{~h}$ at $37^{\circ} \mathrm{C}$ in culture medium with MIF at increasing concentrations from 1 to $1,000 \mathrm{ng} / \mathrm{ml}$. Trypan Blue (Beyotime Institute of Biotechnology, Inc.) was added to the medium, and cells were incubated at $37^{\circ} \mathrm{C}$ for $15 \mathrm{~min}$, phase contrast photomicrographs were taken using an inverted microscope (DMI4000B; Leica, Wetzlar, Germany), and the Trypan Blue-positive cells were counted. Five fields were randomly selected from each culture dish and at least three dishes were counted for each MIF concentration.

Western blot analysis. Western blot analysis was conducted, as previously described (31). Cells were washed twice with ice-cold PBS and lysed with lysis buffer (Beyotime Institute of Biotechnology, Inc.) containing $20 \mathrm{mM}$ Tris- $\mathrm{HCl}, 150 \mathrm{mM}$ $\mathrm{NaCl}, 1 \%$ Triton $\mathrm{X}-100$, and protease and phosphatase inhibitors. Cell extracts were centrifuged for $5 \mathrm{~min}$ at 12,000 $\mathrm{x}$ g and supernatants were collected. Cellular proteins $(20 \mu \mathrm{g})$ were resolved with SDS-PAGE (Beyotime Institute of Biotechnology, Inc.) at $1.5 \mathrm{~mA} / \mathrm{cm}^{2}$ for $90 \mathrm{~min}$ and transferred onto polyvinylidene fluoride membranes (Beyotime Institute of Biotechnology, Inc.). The membranes were blocked for $1 \mathrm{~h}$ with $5 \%$ skimmed milk in Tris-buffered saline containing $0.1 \%$ Tween-20 (Beyotime Institute of Biotechnology, Inc.) and incubated overnight at $4^{\circ} \mathrm{C}$ with the above-mentioned primary antibodies. The membranes were washed and incubated for $1 \mathrm{~h}$ with the appropriate secondary antibodies conjugated to horseradish peroxidase. The membranes were developed using chemiluminescence substrates (Beyotime Institute of Biotechnology, Inc.), photographed with Bio-Rad ChemiDoc XRS equipment (Bio-Rad Laboratories, Inc., Hercules, CA, USA), and quantified and analyzed with Quantity One software (v4.62; Bio-Rad Laboratories, Inc.).

Statistical analysis. Data are expressed as means \pm standard deviations. Differences among groups were assessed by one-way analysis of variance. Comparisons between two groups were evaluated using Student's t-test and $\mathrm{P}<0.05$ was considered to indicate a statistically significant difference.

\section{Results}

MIF protects MSCs from hypoxia/SD-induced apoptosis. Previous studies have indicated that the maximal induction of early apoptosis by hypoxia/SD in MSCs occurs at $24 \mathrm{~h}$ (30). The current study investigated whether MIF protects MSCs from this process. MSCs were incubated with $100 \mathrm{ng} / \mathrm{ml} \mathrm{MIF}$ during exposure to hypoxia/SD for $24 \mathrm{~h}$, and cell apoptosis was then determined by fluorescence-activated cell sorting (Fig. 1). MIF efficiently blocked apoptosis and, compared with the hypoxia/SD cells, the fold increase in the apoptotic index decreased following exposure to $\operatorname{MIF}(3.05 \pm 0.17$ vs. $7.16 \pm 0.35$;
$\mathrm{P}<0.05$; Fig. $1 \mathrm{~A}$ and $\mathrm{C}$ ). To the best of our knowledge, the potential toxicity of MIF to MSCs at these concentrations has not been investigated, thus, the current study examined the effect of increasing concentrations of MIF on MSC viability. Trypan Blue assays indicated that MIF $\leq 1,000 \mathrm{ng} / \mathrm{ml}$ exerted no adverse effect on the viability of MSCs (Fig. 1B and D).

MIF promotes autophagy in MSCs under hypoxia/SD. Previous studies have demonstrated MIF induces autophagy in various cell lines under stress conditions (14). The present study investigated whether MIF, at the concentration that protected MSCs from apoptosis under hypoxia/SD, also promoted autophagy. MIF was observed to promote autophagy, as demonstrated by the conversion of LC3BI to LC3BII (ratio of LC3BII to LC3BI), compared with the hypoxia/SD+MIF cells: $0.82 \pm 0.01$ vs. $0.30 \pm 0.01(\mathrm{P}<0.05$; Fig. $2 \mathrm{~A}$ and $\mathrm{B})$. MIF-induced activation of MSC autophagy was further confirmed by the increased expression level of Atg5 (5.23 \pm 0.20 vs. 1.00 \pm 0.00 ; $\mathrm{P}<0.05$; Fig. 2C and D) and Beclin-1 (3.03 \pm 0.19 vs. $1.00 \pm 0.00$; $\mathrm{P}<0.05$; Fig. 2E and F).

MIF exposure activates the AMPK/mTOR signaling pathway. The AMPK/mTOR signaling pathway is an important regulator of autophagy in multiple cell types $(15,32)$. Therefore, the current study investigated whether this signaling pathway mediates the anti-apoptotic effect of MIF in MSCs (Fig. 3). Western blot analysis revealed low but detectable levels of p-AMPK in control cells, but high levels of p-mTOR. By contrast, MIF exposure induced a marked increase in the ratio of $\mathrm{p}-\mathrm{AMPK}$ to AMPK $(4.97 \pm 0.18$ vs. $1.00 \pm 0.00 ; \mathrm{P}<0.05$; Fig. 3A and $\mathrm{C}$ ), but a decreased ratio of $\mathrm{p}$-mTOR to mTOR $(0.30 \pm 0.02$ vs. $1.00 \pm 0.00 ; \mathrm{P}<0.05$; Fig. $3 \mathrm{~B}$ and $\mathrm{D})$.

Anti-apoptotic effect of MIF is induced by autophagy and mediated by the AMPK/mTOR signaling pathway. Previous studies have identified that autophagy induced by the AMPK/mTOR signaling pathway is important in resistance to apoptosis (15). To investigate whether MIF activates the AMPK/mTOR signaling pathway and induces autophagy to exert an anti-apoptotic effect in MSCs, the AMPK inhibitor, Compound $\mathrm{C}$ or the autophagy inhibitor, 3-MA was administered to MSCs prior to treatment with MIF. Subsequently, the cells were exposed to hypoxia/SD. Apoptosis was measured using flow cytometric assays, which indicated that the fold increase in apoptotic index relative to the control, following hypoxia/SD exposure, was decreased by MIF treatment $(6.70 \pm 0.30$ vs. $3.05 \pm 0.17$; $\mathrm{P}<0.05$; Fig. 4$)$. This effect was reversed by the autophagy inhibitor, 3-MA $(6.81 \pm 0.25$ vs. $3.05 \pm 0.17 ; \mathrm{P}<0.05)$ and by the AMPK inhibitor, Compound $\mathrm{C}(6.79 \pm 0.27$ vs. $3.05 \pm 0.17 ; \mathrm{P}<0.05)$, suggesting that autophagy is induced by the AMPK/mTOR signaling pathway and is involved in the protection of MSCs against hypoxia/SD-induced apoptosis.

\section{Discussion}

Autologous MSCs are effective as therapeutic agents when administered to regenerate the damaged myocardium, and restore heart function following transplantation into an ischemic or infarcted heart. Autologous MSCs are easily 
A

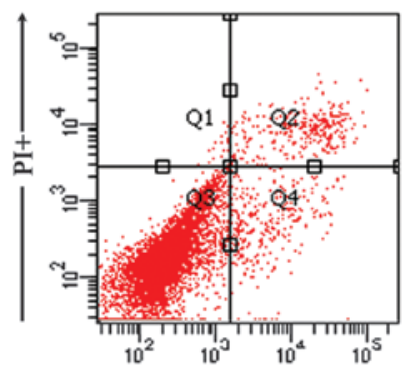

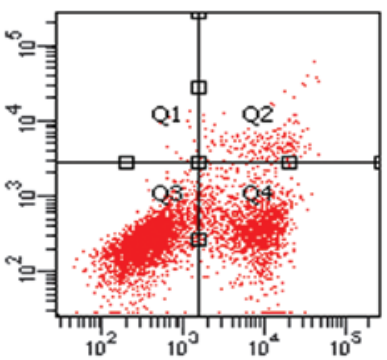

AnnexinV+

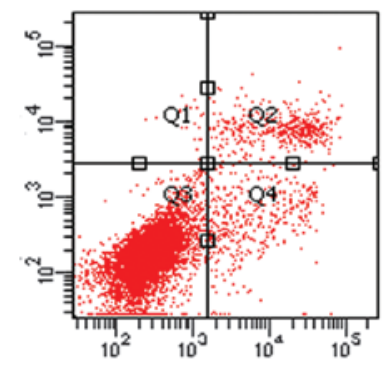

B

$\mathrm{MIF}(\mathrm{mg} / \mathrm{ml})$
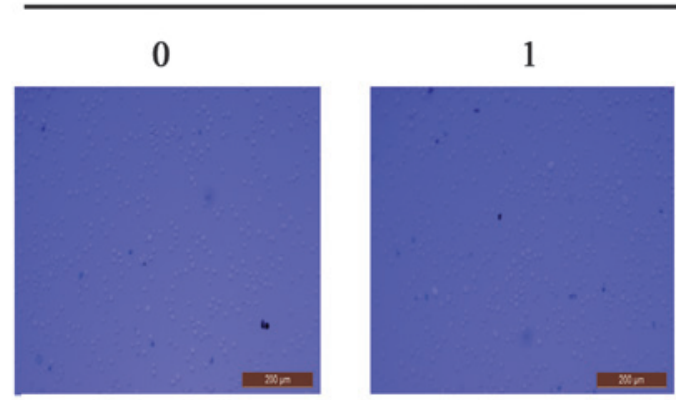

C

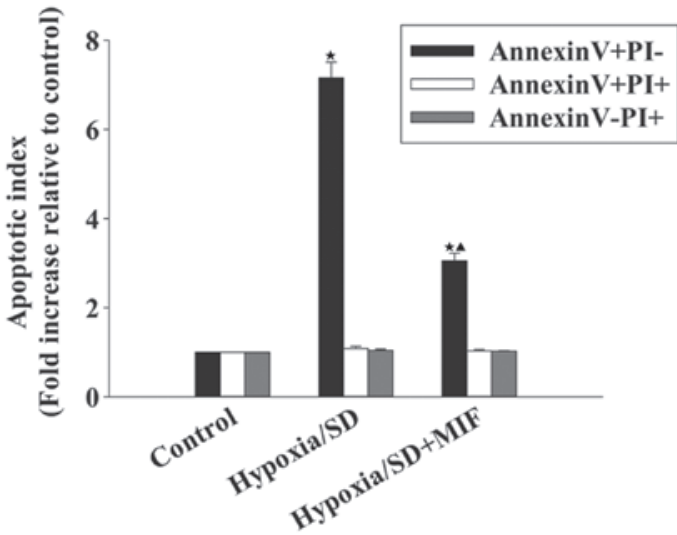

10

100

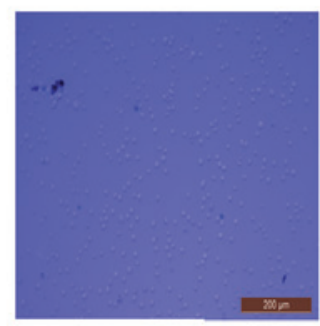

1000
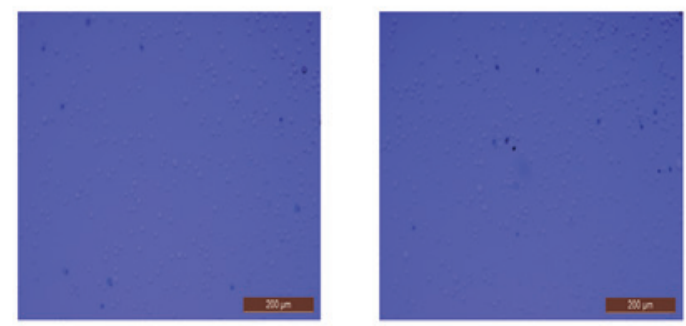

D

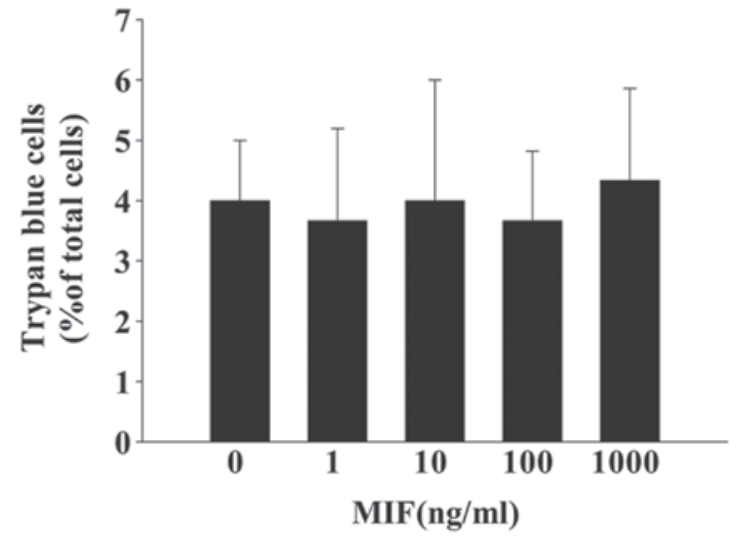

Figure 1. MIF protected MSCs against hypoxia/SD-induced apoptosis. MIF (100 ng/ml) was added to the incubation medium throughout the hypoxia/SD treatment period. (A) Representative images of flow cytometric dot plot analyses of apoptotic cells following Annexin V/PI staining. (B) MIF was added to the culture media alone to investigate its effect on MSC viability. A Trypan Blue assay indicated that MIF $\leq 1,000 \mathrm{ng} / \mathrm{ml}$ was not toxic to cultured MSCs. $(\mathrm{C}$ and $\mathrm{D})$ Each column of the bar chart represents the mean \pm standard deviation of three independent experiments. ${ }^{*} \mathrm{P}<0.05 \mathrm{vs}$. control; ${ }^{\wedge} \mathrm{P}<0.05 \mathrm{vs}$. hypoxia/SD. MIF, macrophage migration inhibitory factor; MSC, mesenchymal stem cell; SD, serum deprivation; PI, propidium iodide.

prepared from adult patients and are immunologically safe (2). However, the extent of MSC engraftment is particularly low despite large numbers of implanted cells, this is primarily due to low cell survival in the ischemic microenvironment (33). Upon transplantation into ischemic tissue, MSCs encounter nutrient and oxygen deprivation and undergo apoptosis; thus, improving MSC survival rates under these conditions is a key issue in MSC-based therapy. In the present study, autophagy induced by MIF is important in protecting MSCs from hypoxia/SD-induced apoptosis via AMPK/mTOR-associated signaling pathways, and may enable MSCs to withstand the growth factor fluctuations and nutrient deprivation that occurs in the ischemic microenvironment, particularly following AMI.

MIF is a pluripotent cytokine that is important in the cellular response to stress (14). MIF contributes to proliferation and survival by preventing cellular apoptosis. Previous studies observed that MIF enhances B cell survival, and demonstrated that MIF promotes cell survival and the proliferation of neural stem cells, suggesting that MIF may be an effective anti-apoptotic agent $(8,13)$. Furthermore, MIF regulates key functions in myocardial ischemia injury and exhibits cardioprotective activity $(12,34,35)$. During myocardial ischemia, MIF modulates the activity of various proteins involved in apoptosis and oxidative stress, including AMPK and mTOR (36). Myocardial MIF may function as an endogenous protection mechanism against ischemic injury; in a previous study of patients with AMI, the serum concentration of MIF was elevated, suggesting that this pathway is active during cardiac ischemia (37). Circulating MIF may provide cardioprotection by elevating energy uptake, but also by protecting cardiomyocytes from apoptosis $(9,12)$. The present study suggests that elevating MIF may enhance MSC survival via activation of pro-survival signaling, and thus may provide cardioprotection during stem cell-based therapy. 
A

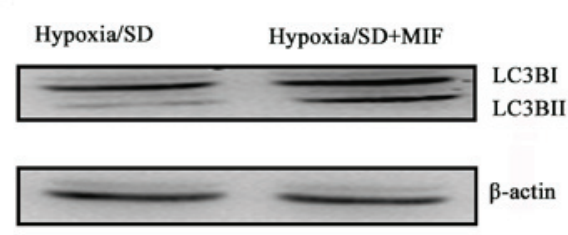

C

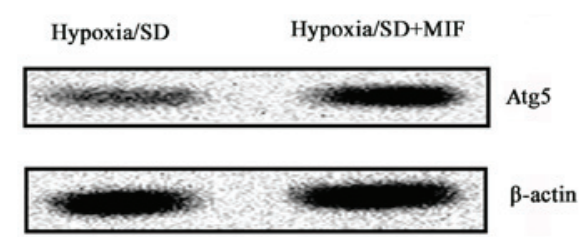

$\mathbf{E}$

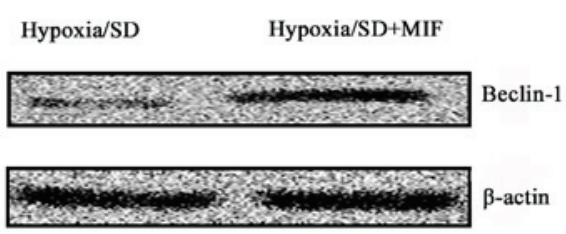

B

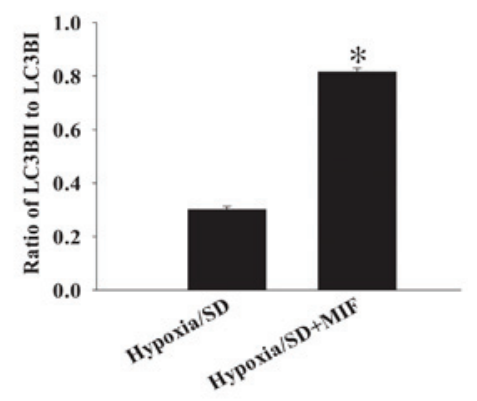

D
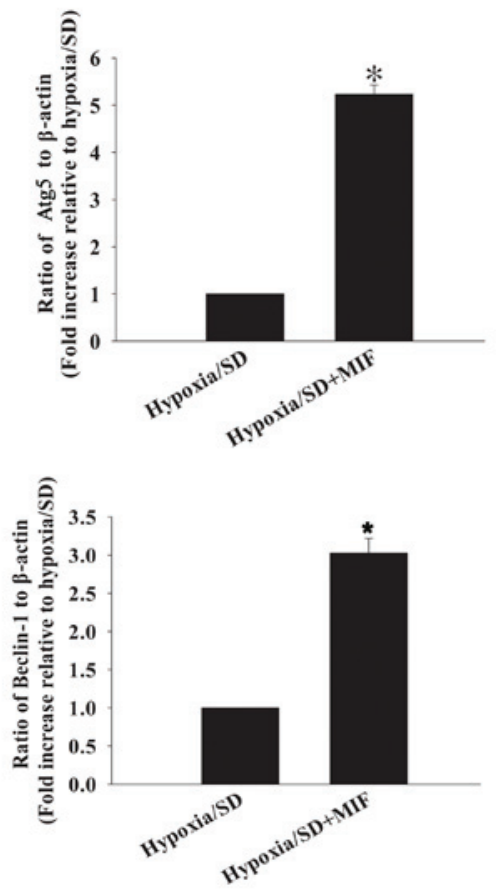

Figure 2. MIF promoted autophagy in MSCs under hypoxia/SD. Western blotting indicated that (A and B) LC3B-II, (C and D) Atg5 and (E and F) Beclin-1 expression levels were increased by MIF under hypoxia/SD. The data represent the results of three independent experiments; ${ }^{*} \mathrm{P}<0.05$ vs. hypoxia/SD group. MIF, macrophage migration inhibitory factor; MSCs, mesenchymal stem cells; SD, serum deprivation; Atg5, autophagy protein 5.

A

Control
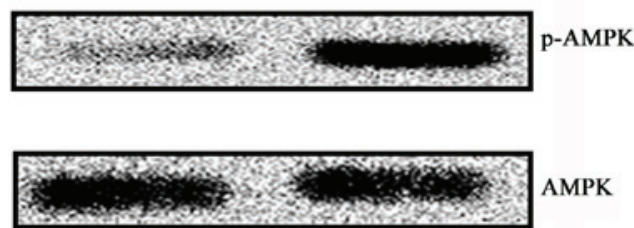

C

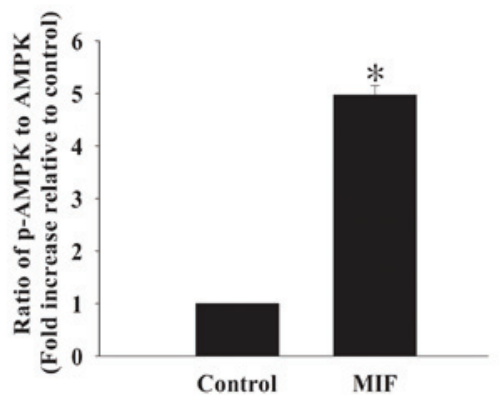

B
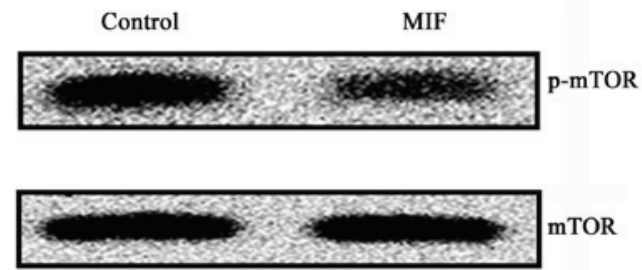

D

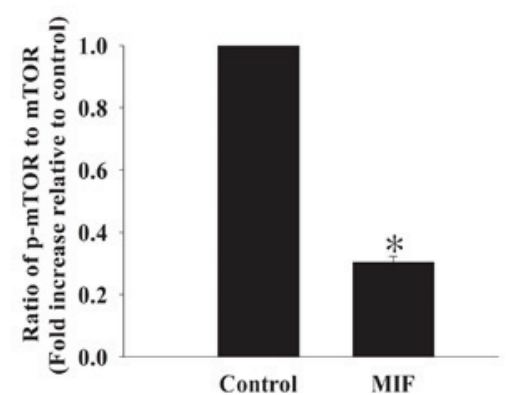

Figure 3. MIF exposure activated the AMPK/mTOR signaling pathway. The AMPK/mTOR signaling pathway in MSCs was examined by western blotting following MIF treatement. Western blot analysis demonstrated that (A) p-AMPK was upregulated by MIF treatment and (B) p-mTOR was downregulated by MIF treatment. (C and D) Bar charts represent the results of three independent experiments; "P $<0.05$ vs. control. MIF, macrophage migration inhibitory factor; AMPK, AMP-activated protein kinase; mTOR, mammalian target of rapamycin; MSC, mesenchymal stem cell; p, phosphorylated. 
A

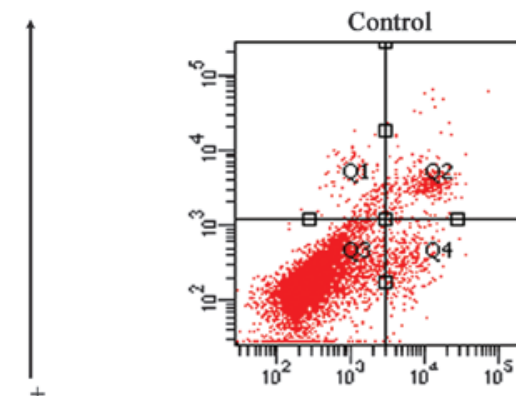

$\frac{ \pm}{2}$

Hypoxia/SD
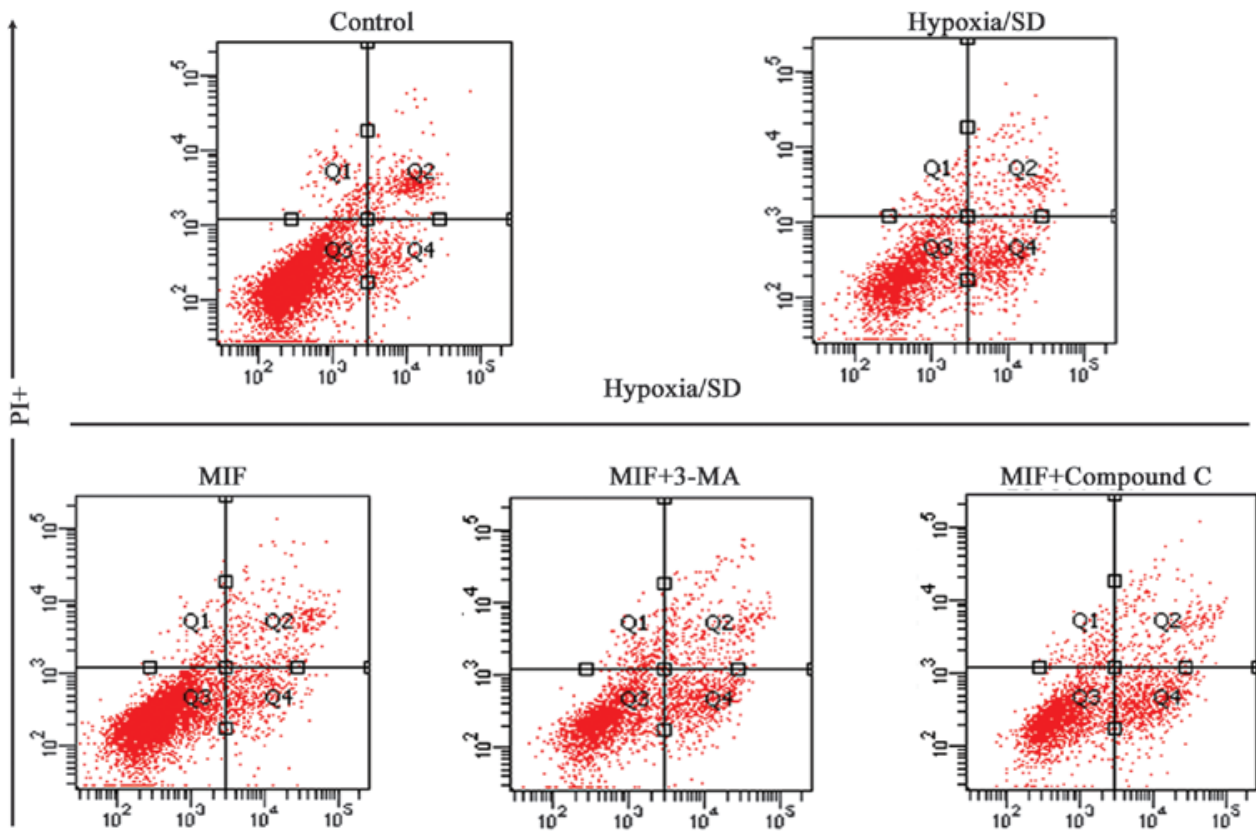

AnnexinV+

B

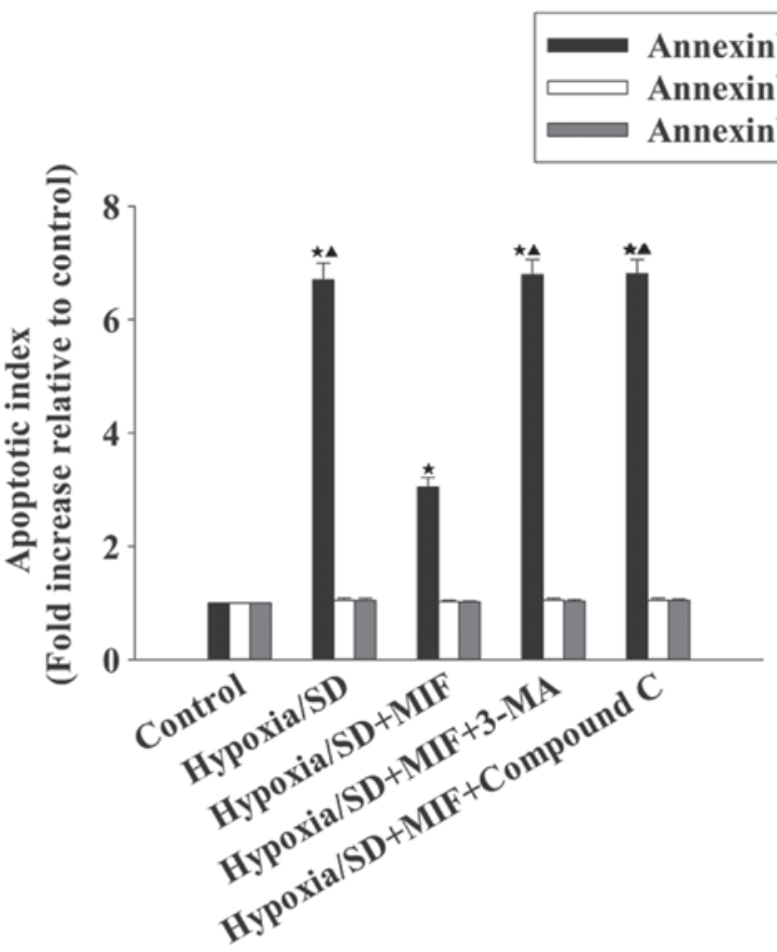

Figure 4. Anti-apoptotic effect of MIF was induced by autophagy via the AMPK/mTOR signaling pathway. MSCs were pretreated with autophagy inhibitor 3-MA $(5 \mu \mathrm{M})$ or AMPK inhibitor, Compound $\mathrm{C}(10 \mu \mathrm{M})$ for $90 \mathrm{~min}$ in complete medium prior to exposure to hypoxia/SD. MIF (100 ng/ml) was added in the presence of the inhibitor at the beginning of hypoxia/SD exposure. All therapeutic agents were maintained in the incubation medium throughout the hypoxia/SD treatment period. Apoptosis was quantified by flow cytometry. (A) Representative images of flow cytometric dot plot analyses of apoptotic cells following Annexin V/PI staining. (B) Composite values of the apoptotic index presented as fold change from the control. Each column represents the mean \pm standard deviation of three independent experiments; ${ }^{*} \mathrm{P}<0.05$ vs. control; $\triangle \mathrm{P}<0.05$ vs. hypoxia/SD $+\mathrm{MIF}$. MIF, macrophage migration inhibitory factor; AMPK, AMP-activated protein kinase; mTOR, mammalian target of rapamycin; MSC, mesenchymal stem cell; SD, serum deprivation; PI, propidium iodide.

Decreased cell survival under ischemic stress is an obstacle for stem cell-based therapy. One cellular response, which is critical for cell survival under metabolic stress and energy starvation, is autophagy, which is a catabolic process that delivers cytoplasmic components to lysosomes for degradation (38). Hypoxia and SD are energy-limiting stressors and, under these conditions, adaptive autophagy is essential for cell survival by increasing cellular energy supply, and eliminating damaged organelles and free radicals $(39,40)$. Recently MIF has been implicated in the control of autophagy as a regulator of cellular fate (9). Under stress conditions, MIF induced autophagy to maintain heart function and protect cardiomyocytes from 
apoptosis (9). Similarly, in the present study, MIF protected MSCs from apoptosis, which was accompanied by an increase in autophagy. The protective effect of MIF was eliminated by the autophagy inhibitor, 3-MA, confirming that MIF exerted its anti-apoptotic effect by modulating autophagy in MSCs.

AMPK is a stress-signaling kinase, and a key regulator of energy generation and consumption pathways, which protect cells against hypoxic injury and cell death $(32,41)$. The release of endogenous MIF from ischemic myocardium has been demonstrated to stimulate AMPK activation in a paracrine manner, leading to enhanced glucose uptake and a beneficial tissue-protective response (12). A major mechanism underlying the effect of AMPK is the modulation of autophagy under stress conditions (9). During hypoxia/SD or other energy shortage situations, AMPK may act as a sensor of cellular energy change and become activated by a decreased ATP/AMP ratio $(32,41)$. In addition, mTOR, a major downstream target of AMPK, stimulates autophagy by inactivating mTOR complex 1, an inhibitor of the autophagy pathway (42). Thus, the AMPK/mTOR signaling pathway is hypothesized to be a positive regulator of autophagy during hypoxia, starvation, or other energy stress events $(14,42)$. The current study indicated that MIF increased AMPK phosphorylation and decreased mTOR phosphorylation, while inhibition of AMPK blocked the anti-apoptotic effect of MIF. These results suggest that the AMPK/mTOR signaling pathway serves as a potential underlying mechanism by which MIF promotes autophagy in MSCs to resist hypoxia/SD-induced apoptosis.

In conclusion, MIF may be able to promote MSC survival under conditions that mimic the ischemic myocardium. The results of the current study suggest that MIF protects MSCs from hypoxia/SD-induced apoptosis by regulating autophagy via the AMPK/mTOR signaling pathway. These results highlight potential novel therapeutic strategies for protecting MSCs from apoptosis, and provide a basis for the clinical application of MIF and MSCs in cardiac regeneration therapeutic strategies.

\section{References}

1. Segers VF and Lee RT: Stem-cell therapy for cardiac disease. Nature 451: 937-942, 2008

2. Nesselmann C, Ma N, Bieback K, Wagner W,Ho A, Konttinen YT, Zhang H, Hinescu ME and Steinhoff G: Mesenchymal stem cells and cardiac repair. J Cell Mol Med 12 (5B): 1795-1810, 2008.

3. Li RK, Mickle DA, Weisel RD, Rao V and Jia ZQ: Optimal time for cardiomyocyte transplantation to maximize myocardial function after left ventricular injury. Ann Thorac Surg 72: 1957-1963, 2001.

4. Zhu W, Chen J, Cong X, Hu S and Chen X: Hypoxia and serum deprivation-induced apoptosis in mesenchymal stem cells. Stem Cells 24: 416-425, 2006.

5. Burger-Kentischer A, Goebel H, Seiler R, Fraedrich G, Schaefer HE, Dimmeler S, Kleeman R, Bernhagen J and Ihling C: Expression of macrophage migration inhibitory factor in different stages of human atherosclerosis. Circulation 105: 1561-1566, 2002.

6. Calandra T, Bernhagen J, Mitchell RA and Bucala R: The macrophage is an important and previously unrecognized source of macrophage migration inhibitory factor. J Exp Med 179: 1895-1902, 1994.

7. Willis MS, Carlson DL, Dimaio JM, White MD, White DJ, Adams GA IV, Horton JW and Giroir BP: Macrophage migration inhibitory factor mediates late cardiac dysfunction after burn injury. Am J Physiol Heart Circ Physiol 288: H795-H804, 2005.

8. Ohta S, Misawa A, Fukaya R, Inoue S, Kanemura Y, Okano H, Kawakami Y and Toda M: Macrophage migration inhibitory factor (MIF) promotes cell survival and proliferation of neural stem/progenitor cells. J Cell Sci 125: 3210-3220, 2012.
9. Xu X, Pacheco BD, Leng L, Bucala R and Ren J: Macrophage migration inhibitory factor plays a permissive role in the maintenance of cardiac contractile function under starvation through regulation of autophagy. Cardiovasc Res 99: 412-421, 2013.

10. Calandra T, Bernhagen J, Metz CN,Spiegel LA, Bacher M,Donnelly T, Cerami A and Bucala R: MIF as a glucocorticoid-induced modulator of cytokine production. Nature 377: 68-71, 1995.

11. Benigni F, Atsumi T, Calandra T, Metz C, Echtenacher B, Peng T and Bucala R: The proinflammatory mediator macrophage migration inhibitory factor induces glucose catabolism in muscle. J Clin Invest 106: 1291-1300, 2000.

12. Miller EJ, Li J, Leng L, McDonald C, Atsumi T, Bucala R and Young LH: Macrophage migration inhibitory factor stimulates AMP-activated protein kinase in the ischaemic heart. Nature 451: 578-582, 2008.

13. Gore Y, Starlets D, Maharshak N, Becker-Herman S, Kaneyuki U, Leng L, Bucala R and Shachar I: Macrophage migration inhibitory factor induces B cell survival by activation of a CD74-CD44 receptor complex. J Biol Chem 283: 2784-2792, 2008.

14. Chuang YC, Su WH, Lei HY, Lin YS, Liu HS, Chang CP and Yeh TM: Macrophage migration inhibitory factor induces autophagy via reactive oxygen species generation. PLoS One 7: e37613, 2012.

15. Zhang Q, Yang YJ, Wang H, Dong QT, Wang TJ, Qian HY and $\mathrm{Xu} \mathrm{H}$ : Autophagy activation: A novel mechanism of atorvastatin to protect mesenchymal stem cells from hypoxia and serum deprivation via AMP-activated protein kinase/mammalian target of rapamycin pathway. Stem Cells Dev 21: 1321-1332, 2012.

16. Mizushima N: Autophagy: Process and function. Genes Dev 21: 2861-2873, 2007.

17. Yang Z and Klionsky DJ: Mammalian autophagy: Core molecular machinery and signaling regulation. Curr Opin Cell Biol 22: 124-131, 2010.

18. Ferraro E and Cecconi F: Autophagic and apoptotic response to stress signals in mammalian cells. Arch Biochem Biophys 462: 210-219, 2007.

19. He C and Klionsky DJ: Regulation mechanisms and signaling pathways of autophagy. Annu Rev Genet 43: 67-93, 2009.

20. Danial NN and Korsmeyer SJ: Cell death: Critical control points. Cell 116: 205-219, 2004.

21. Baldwin AS: Regulation of cell death and autophagy by IKK and NF- $\kappa$ B: critical mechanisms in immune function and cancer. Immunol Rev 246: 327-345, 2012.

22. Nunnari J and Suomalainen A: Mitochondria: in sickness and in health. Cell 148: 1145-1159, 2012

23. Debnath J, Baehrecke EH and Kroemer G: Does autophagy contribute to cell death? Autophagy 1: 66-74, 2005.

24. Maiuri MC, Zalckvar E, Kimchi A and Kroemer G: Self-eating and self-killing: Crosstalk between autophagy and apoptosis. Nat Rev Mol Cell Biol 8: 741-752, 2007.

25. Herberg S, Shi X, Johnson MH, Hamrick MW, Isales CM and Hill WD: Stromal cell-derived factor-1 $\beta$ mediates cell survival through enhancing autophagy in bone marrow-derived mesenchymal stem cells. PLoS One 8: e58207, 2013.

26. Nepal S and Park PH: Activation of autophagy by globular adiponectin attenuates ethanol-induced apoptosis in HepG2 cells: Involvement of AMPK/FoxO3A axis. Biochim Biophys Acta 1833: 2111-2125, 2013

27. Warr MR, Binnewies M, Flach J, Reynaud D, Garg T, Malhotra R, Debnath $\mathrm{J}$ and Passegué E: FOXO3A directs a protective autophagy program in haematopoietic stem cells. Nature 494: 323-327, 2013.

28. Zhang Z, Li S, Cui M, Gao X, Sun D, Qin X, Narsinh K, Li C, Jia H, Li C, et al: Rosuvastatin enhances the therapeutic efficacy of adipose-derived mesenchymal stem cells for myocardial infarction via PI3K/Akt and MEK/ERK pathways. Basic Res Cardiol 108: 333, 2013.

29. Liu WH, Liu JJ, Wu J, Zhang LL, Liu F, Yin L, Zhang MM and Yu B: Novel mechanism of inhibition of dendritic cells maturation by mesenchymal stem cells via interleukin-10 and the JAK1/STAT3 sinaling pathway. PLoS One 8: e55487, 2013.

30. Hou M, Cui J, Liu J, Liu F, Jiang R, Liu K, Wang Y, Yin L, Liu W and Yu B: Angiopoietin-like 4 confers resistance to hypoxia/serum deprivation-induced apoptosis through PI3K/Akt and ERK1/2 signaling pathways in mesenchymal stem cells. PloS one 9: e85808, 2014.

31. Chen J, Baydoun AR, Xu R, Deng L, Liu X, Zhu W, Shi L, Cong X, $\mathrm{Hu} \mathrm{S}$ and Chen X: Lysophosphatidic acid protects mesenchymal stem cells against hypoxia and serum deprivation-induced apoptosis. Stem Cells 26: 135-145, 2008. 
32. Hardie DG, Ross FA and Hawley SA: AMPK: A nutrient and energy sensor that maintains energy homeostasis. Nat Rev Mol Cell Biol 13: 251-262, 2012.

33. Mangi AA, Noiseux N, Kong D, He H, Rezvani M, Ingwall JS and Dzau VJ: Mesenchymal stem cells modified with Akt prevent remodeling and restore performance of infarcted hearts. Nat Med 9: 1195-1201, 2003.

34. Koga K, Kenessey A, Powell SR, Sison CP, Miller EJ and Ojamaa K: Macrophage migration inhibitory factor provides cardioprotection during ischemia/reperfusion by reducing oxidative stress. Antioxid Redox Signal 14: 1191-1202, 2011.

35. Ma H, Wang J, Thomas DP, Tong C, Leng L, Wang W, Merk M, Zierow S, Bernhagen J, Ren J, et al: Impaired macrophage migration inhibitory factor-AMP-activated protein kinase activation and ischemic recovery in the senescent heart. Circulation 122: 282-292, 2010

36. Haldar SM and Stamler JS: S-nitrosylation: Integrator of cardiovascular performance and oxygen delivery. J Clin Invest 123 101-110, 2013.

37. Takahashi M, Nishihira J, Shimpo M, Mizue Y, Ueno S, Mano H, Kobayashi E, Ikeda U and Shimada K: Macrophage migration inhibitory factor as a redox-sensitive cytokine in cardiac myocytes. Cardiovasc Res 52: 438-445, 2001.
38. Qiang L, Wu C, Ming M, Viollet B and He YY: Autophagy controls p38 activation to promote cell survival under genotoxic stress. J Biol Chem 288: 1603-1611, 2013.

39. Zhang H, Bosch-Marce M, Shimoda LA, Tan YS, Baek JH, Wesley JB, Gonzalez FJ and Semenza GL: Mitochondrial autophagy is an HIF-1-dependent adaptive metabolic response to hypoxia. J Biol Chem 283: 10892-10903, 2008.

40. Hamacher-Brady A, Brady NR, Logue SE, Sayen MR, Jinno M, Kirshenbaum LA, Gottlieb RA and Gustafsson $\mathrm{AB}$ : Response to myocardial ischemia/reperfusion injury involves Bnip3 and autophagy. Cell Death Differ 14: 146-157, 2007.

41. Nagendran J, Waller TJ and Dyck JR: AMPK signalling and the control of substrate use in the heart. Mol Cell Endocrinol 366: 180-193, 2013.

42. Kapahi P, Chen D, Rogers AN, Katewa SD, Li PW, Thomas EL and Kockel L: With TOR, less is more: A key role for the conserved nutrient-sensing TOR pathway in aging. Cell Metab 11: 453-465, 2010 